\title{
Structural basis of EB1 effects on microtubule dynamics
}

Frédéric M. Coquelle ${ }^{1}$, Benjamin Vitre and Isabelle Arnal ${ }^{1}$

Université de Rennes 1, Interactions Cellulaires et Moléculaires, Centre National de la Recherche Scientifique, Unité Mixte de Recherche 6026,

Institut Fédératif de Recherche 140, Génétique Fonctionnelle Agronomie et Santé, Campus de Beaulieu,

263, Avenue du Général Leclerc,

35042 Rennes,

France

${ }^{1}$ Correspondence may be addressed to either of these authors (Email isabelle.arnal@univ-rennes1.fr or frederic.coquelle@univ-rennes1.fr).

Key words: microtubule, MAPs, +TIPs, EB1, dynamic instability, tubulin sheet.

Abbreviations: +TIPs (+end Tracking Proteins), EB1 (End-Binding 1), CryoEM (Electron CryoMicroscopy), MAPs (Microtubule Associated Proteins), TIRF (Total Internal Reflection Fluorescence), VE-DIC (Video-Enhanced Differential Interference Contrast).

\begin{abstract}
Plus-end tracking proteins (+TIPs) are an increasing group of molecules that localize preferentially to the end of growing microtubules. +TIPs regulate microtubule dynamics and contribute to the organization of the microtubular network within the cell. Thus, they participate in a wide range of cellular processes including cell division, motility and morphogenesis. EB1 (End-Binding 1) is a highly conserved key member of the +TIP group that has been shown to modulate microtubule dynamicity both in vitro and in cells. EB1 is involved in the accurate chromosome segregation during mitosis and in the polarization of the microtubule cytoskeleton in migrating cells. Here, we review recent in vitro studies that have started to reveal a regulating activity of EB1, and its yeast ortholog Mal3p, on microtubule structure. In particular, we examine how EB1-mediated changes in the microtubule architecture may explain its effects on microtubule dynamics.
\end{abstract}




\section{The microtubule, a dynamic structure.}

Microtubules are polar and dynamic polymers involved in the functional organization of most eukaryotic cells. They are hollow cylinders about $25 \mathrm{~nm}$ in diameter resulting from the assembly of $\alpha \beta$-tubulin heterodimers [1]. Tubulin dimers are arranged head-to-tail to form protofilaments that interact laterally making the cylindrical microtubule wall (Figure 1A). This particular arrangement confers to the microtubule a structural polarity with the $\beta$-subunit exposed at the fastest-growing plus end [2]. Protofilaments are longitudinally shifted from one to the other, so that tubulin monomers describe left-handed lateral helices within the microtubule lattice. The architecture of a classical cytoplasmic microtubule consists of 13 protofilaments aligned parallel to the tube axis and three lateral helices (denoted hereafter: 13_3 microtubule, Figure 1A) [3]. All other protofilament and/or lateral helix number configurations exhibit a small twist of the protofilaments, so as the same helical geometry found in the 13_3 microtubule is maintained (Figure 1B) [4,5]. As a result of this protofilament skewing, electron micrographs of vitreous-ice embedded microtubules display characteristic moiré patterns that allow to accurately determine the surface-lattice organization and microtubule polarity [5-8]. Protofilament skewing and changes in the microtubule radial curvature have been proposed to induce the accumulation of an excess of energy in the polymer, capable of destabilizing interactions between tubulin dimers [9, 10]. As a consequence, unskewed 13_3 microtubules, which represent the most common in vivo architecture, would also be the most energetically favorable ones.

Other remarkable structural features have been characterized at the end of microtubules, which are clearly distinguishable from the tube. Outwardly curled, separated protofilaments appear at the depolymerizing microtubule extremity [11-14], whereas a two-dimensional sheet of tubulin has been observed at the end of growing microtubules in vitro [11, 12, 15-18] (Figure 1D) and in cells [19-23]. These observations suggested that microtubule assembly is a two-dimensional process occurring through the extension of an outwardly curved tubulin sheet that gradually closes into a tube [24]. According to the proposed model, sheets display two opposite curvatures resulting from (1) the longitudinal intrinsic curvature of protofilaments and (2) the lateral interactions between protofilaments (green and blue arrows respectively on Figure 1C, reviewed in details in [29]). The latter increases with the number of protofilaments, which leads to the gradual straightening and closure of the sheet into a cylinder. Hence, the closure of the sheet is intimately linked to the balance between its lateral and longitudinal growth. This 2D-assembly model also implies that the lattice organization is determined by the tubulin-sheet closure, unlike the template model based on microtubule helical growth [25]. 
Microtubules undergo dynamic instability, an intrinsic property characterized by stochastic transitions between growing and shrinking states [1]. This striking behavior, which is essential for diverse cellular processes, is characterized by the following four parameters: the growth rate, the shrinkage rate, the catastrophe frequency (transition from growth to shrinkage) and the rescue frequency (reverse transition). Despite extensive research in the field, the molecular mechanisms sustaining these random transitions are still widely unknown, but models have been developed. Dynamic instability is fueled by GTP hydrolysis on $\beta$-tubulins, which led primarily to the model of the so-called GTP-cap [26]. This cap of GTP-bound $\beta$-tubulin was proposed to protect microtubules from catastrophes by reinforcing lateral and/or longitudinal tubulin interactions at microtubule ends $[27,28]$. Later on, the presence of outwardly curved tubulin sheets at the end of growing microtubules led to the conformational cap model [29]. Tubulin-sheet closure compels protofilaments to straighten up, accumulating elastic energy into the lattice. As a consequence, the tubulin sheet appears more stable than the cylindrical wall, and would therefore constitute a protective cap at microtubule ends. Recent structural studies and computed modelization support the relevance of such a conformational cap [24, 30-32]. Today, the relationship between the conformational cap and GTP hydrolysis is not clearly understood, even though a conformational antibody raised against GTP-bound tubulin has revealed the end of growing microtubules in immunostaining experiments [33]. Whether this antibody recognizes the curved conformation of tubulin present in two-dimensional sheets remains to be determined. One possibility would be that GTP hydrolysis is triggered by the tubulin sheet straightening, making a rough overlap between the sheet and the GTP-cap, as suggested elsewhere [29].

Dynamic instability is modulated within the cell by a myriad of MAPs (Microtubule Associated Proteins) [34]. Their effects on microtubule dynamics and polarization have been well described in the past two decades, but their precise molecular mechanisms are still unknown. Nonetheless, recent concepts have started to rise concerning the effects of MAPs on microtubule structure, including lattice architecture and tubulin-sheet morphology. As an example, the neuronal MAP doublecortin seems to force specifically the assembly of 13_3 microtubules while modulating microtubule dynamics in vitro $[35,36]$. Thus, the current challenge consists of deciphering the link between the control of microtubule structure and the regulation of microtubule dynamics.

\section{EB1, a plus-end tracking protein (+TIP) that tunes microtubule dynamics.}

+TIPs are the most recently described group of MAPs that localize preferentially to the growing microtubule end, where they constitute an intricate molecular network [37]. +TIPs play 
fundamental roles in the control of microtubule dynamics and polarization during the cell cycle, so as they participate in diverse cellular processes, like cell division, migration and differentiation [38]. EB1 is a highly conserved key member of the +TIP group. First described as an interactor of the tumor suppressor APC, it is now better known for being the "key stone" of the +TIP network [37, 39-41]. EB1 tracks microtubule ends by treadmilling at the end of microtubules. This process, which has been recently reconstituted in vitro with purified components [40-42], is characterized by the association of the protein with the newly growing end coupled with a rapid dissociation from the lattice older part. The recognition of a specific structure at microtubule plus end, like the tubulin sheet, is the favorite targeting mechanism, rather than a copolymerization with tubulin heterodimers, since EB1 has a weak affinity for the latter [43-46]. Furthermore, the dwell time of EB1 at microtubule ends is too brief to account for the whole comet decoration through a copolymerization process [40, 41]. Afterwards, EB1 could come unstuck from the microtubule lattice following the conformational change induced by tubulin sheet closure. Phosphorylation and/or interaction with other factors could influence this behavior in vivo. GTP hydrolysis might also be involved in EB1 tracking, even if it does not modify drastically the $\alpha \beta$-heterodimer conformation [30-32]. Further experiments, including precise EB1 localization by cryo-electron tomography, will be necessary to superimpose clearly EB1 comets observed in fluorescence microscopy with tubulin sheets. However, a recent in vitro study, based on TIRF experiments, has revealed that the mean comet length increases with the microtubule growth rate [40] as shown before for the tubulin sheet [12].

It is now clear that EB1 has strong effects on microtubule dynamics in vitro and in vivo, but its precise activity on each parameter of dynamic instability is still contradictory and controversial in the literature. Firstly, EB1 has been shown to stimulate both catastrophes and rescues in Saccharomyces cerevisiae and Drosophila melanogaster [47, 48], while it inhibits catastrophes in Schizosaccharomyces pombe, Xenopus laevis egg extracts, and cultured mammalian cells [43, 49, 50]. Secondly, based on in vitro turbidimetry assays, several studies have revealed that EB1 is able to promote, on its own, tubulin assembly [42, 45, 51-53], whereas others have suggested the need of an additional +TIP to get the same activity [46, 54, 55]. Finally, the use of video-enhanced differential interference contrast (VE-DIC) light microscopy, which allows to record individual microtubule behavior, did not clarify much our understanding regarding the precise effects of EB1 on microtubule dynamics. Indeed, in two studies, EB1 has been shown to strongly increase the microtubule dynamicity [42, 53], whereas in another one it decreases catastrophes [52]. Furthermore, a very recent report has demonstrated that EB3, another member of the conserved EB1 protein family, promotes microtuble dynamics in vitro [50]. These discrepancies among all these studies may be explained by differences in the complementary DNA employed (e.g. Human- 
versus Mouse-EB1) and the experimental conditions used, including variable EB1:tubulin ratios and diverse microtubule assembly buffers. As an example, assembly buffers may contain $\mathrm{KCl}$ or $\mathrm{NaCl}$ at various concentrations, while these salts are known to affect not only the ionic strength, but also the microtubule assembly per se [56] (and unpublished data). In addition, VE-DIC studies have been performed under conditions in which the concentration of free tubulin heterodimers either remained roughly constant $[42,53]$ or decreased progressively during the experiment [52].

\section{EB1 shapes microtubule lattice during tubulin sheet closure.}

Structural studies have been recently performed in order to decipher in details the molecular mechanisms sustaining EB1/Mal3p activity on microtubule dynamics [45, 53, 57].

Using high-resolution surface shadowing and electron microscopy, Mal3p has been first localized along the microtubule seam, which is thought to be the resulting tube junction area. Consistent with the previous analysis, a three-dimensional reconstruction based on cryo-electron microscopy images revealed that the amino-terminal part of Mal3p closely binds to tubulin monomer on the outer surface of microtubules and occupies the groove between protofilaments. Overall, these primary structural data strongly suggest that Mal3p would reinforce the lateral interactions between adjacent protofilaments, in particular at the microtubule closure junction. This lattice-based activity may stabilize the microtubule wall, and thereby account for the stimulating effects of EB1/Mal3p on rescue events [53]. It is worth noting that the stimulation of rescues by EB1, unlike its other effects on microtubule dynamics, has been observed only for the higher concentrations used in in vitro assembly experiments [53]. This is consistent with the very low affinity of EB1/Mal3p for the microtubule lattice $[40,45,52]$, and suggests that the other modulating activities of this +TIP on microtubule dynamics are based on its preferential binding to microtubule ends. However, recent publications favor a solely lattice based activity to explain the diverse effects of EB1 on dynamic instability $[40,41]$. Hence, further experiments will be needed to clearly discriminate between the lattice-based activities of EB1 and the ones that are confined to microtubule extremities.

Microtubules assembled in vitro display a reduced mean sheet length at their extremities and a lower overall proportion of tubulin sheet in the presence of either Mal3p or EB1 [45, 53]. Given the localization of Mal3p on microtubule seams, it has been primarily proposed that this +TIP may process sheet closure through a zippering activity (Figure 2A) [45]. Alternatively, EB1 may bind preferentially to the edges of tubulin sheet where it could facilitate the incorporation of tubulin heterodimers and/or oligomers [53]. Through this mechanism, EB1 would increase the tubulin-sheet lateral curvature, resulting in their straightening up and closure into a tube (as described in Figure 1C). Both models may account for EB1 stimulating effects on the catastrophe frequency, since microtubules with a shorter conformational cap are thought to be more prompt to undergo a 
depolymerization event [12]. However, only the second model may explain the EB1-mediated stimulation of the microtubule growth rate by the increase of the incoming tubulin flux.

EB1 and Mal3p favor the unskewed 13_3 lattice architecture in co-assembly experiments [53, 57]. Such an effect has been already observed with doublecortin and was attributed to a constraining activity [35, 36]. Since Mal3p localizes, like doublecortin, in between protofilaments, it has been proposed that EB1/Mal3p forces the 13_3 configuration by controlling the microtubule radial curvature [57]. Nevertheless, unlike doublecortin, EB1/Mal3p preferentially localizes to microtubule ends, suggesting that its constraining activity would be confined around the microtubule closure junction (Figure 2B). Interestingly, this eventual modus operandi does not explain how EB1 stimulates the catastrophe frequency and the growth rate, which opens the possibility of two independent EB1-mediated mechanisms at microtubule ends.

In parallel, based on the EB1/Mal3p sheet closing activity, a more indirect mechanism has been put forward to explain the selective accumulation of 13_3 microtubules [53]. Indeed, EB1/Mal3p mediated reduction of the conformational cap destabilizes microtubules, leading to the elimination, at first, of the more unstable lattice configurations through catastrophe events, as proposed before [10]. Thus, this phenomenon is thought to contribute indirectly to the selection of 13_3 microtubules by discarding the more stressed configurations over the time (Figure 2A). Additionally, a more direct proofreading activity has been considered that remains to be demonstrated [53]. In all likelihood, this mechanism would be localized close to the tube junction area where EB1/Mal3p would be able to detect, as a sensor, any lattice defect and trigger a catastrophe event to eliminate it (Figure 2B). The localization of Mal3p in the groove between protofilaments [57] is compatible with a radial curvature sensor activity, but further investigations are required to shed light into the intimate molecular mechanisms involved. Three-dimensional models combining electron microscope and X-ray crystallography data represent promising future directions to identify, at the atomic level, the molecular interactions in play between EB1 and the microtubule.

\section{Acknowledgments:}

We thank the National Center for Scientific Research (CNRS), the French Ministry of Research, the National Research Agency (ANR) and the Association pour la Recherche sur le Cancer (ARC) for support. 


\section{References:}

1 Desai, A. and Mitchison, T. J. (1997) Microtubule polymerization dynamics. Annu. Rev. Cell Dev. Biol. 13, 83-117

2 Nogales, E. (1999) A structural view of microtubule dynamics. Cell. Mol. Life. Sci. 56, 133142

3 Amos, L. and Klug, A. (1974) Arrangement of subunits in flagellar microtubules. J. Cell Sci. 14, 523-549

4 Wade, R. H., Chrétien, D. and Job, D. (1990) Characterization of microtubule protofilament numbers. How does the surface lattice accommodate? J. Mol. Biol. 212, 775-786

5 Chrétien, D. and Wade, R. H. (1991) New data on the microtubule surface lattice. Biology of the cell / under the auspices of the European Cell Biology Organization 71, 161-174

6 Mandelkow, E. M. and Mandelkow, E. (1985) Unstained microtubules studied by cryoelectron microscopy. Substructure, supertwist and disassembly. J. Mol. Biol. 181, 123-135

7 Mandelkow, E. and Mandelkow, E. M. (1986) Quick-frozen microtubules studied by cryoelectron microscopy and image processing. Ann. N. Y. Acad. Sci. 483, 13-23

8 Chrétien, D., Kenney, J. M., Fuller, S. D. and Wade, R. H. (1996) Determination of microtubule polarity by cryo-electron microscopy. Structure (London, England) 4, 10311040

9 Chrétien, D. and Fuller, S. D. (2000) Microtubules switch occasionally into unfavorable configurations during elongation. J. Mol. Biol. 298, 663-676

10 Hunyadi, V., Chrétien, D. and Jánosi, I. M. (2005) Mechanical stress induced mechanism of microtubule catastrophes. J. Mol. Biol. 348, 927-938

11 Mandelkow, E. M., Mandelkow, E. and Milligan, R. A. (1991) Microtubule dynamics and microtubule caps: a time-resolved cryo-electron microscopy study. J. Cell Biol. 114, 977991.

12 Chrétien, D., Fuller, S. D. and Karsenti, E. (1995) Structure of growing microtubule ends: two-dimensional sheets close into tubes at variable rates. J. Cell Biol. 129, 1311-1328.

13 Tran, P. T., Joshi, P. and Salmon, E. D. (1997) How tubulin subunits are lost from the shortening ends of microtubules. J. Struct. Biol. 118, 107-118

14 Muller-Reichert, T., Chretien, D., Severin, F. and Hyman, A. A. (1998) Structural changes at microtubule ends accompanying GTP hydrolysis: information from a slowly hydrolyzable analogue of GTP, guanylyl (alpha,beta)methylenediphosphonate. Proc. Natl. Acad. Sci. U. S. A. 95, 3661-3666

15 Erickson, H. P. (1974) Microtubule surface lattice and subunit structure and observations on reassembly. J. Cell Biol. 60, 153-167

16 Kirschner, M. W., Williams, R. C., Weingarten, M. and Gerhart, J. C. (1974) Microtubules from mammalian brain: some properties of their depolymerization products and a proposed mechanism of assembly and disassembly. Proc. Natl. Acad. Sci. U. S. A. 71, 1159-1163

17 Simon, J. R. and Salmon, E. D. (1990) The structure of microtubule ends during the elongation and shortening phases of dynamic instability examined by negative-stain electron microscopy. J. Cell Sci. 96, 571-582.

18 Arnal, I., Karsenti, E. and Hyman, A. A. (2000) Structural transitions at microtubule ends correlate with their dynamic properties in Xenopus egg extracts. J. Cell Biol. 149, 767-774.

19 O'Toole, E. T., McDonald, K. L., Mantler, J., McIntosh, J. R., Hyman, A. A. and MullerReichert, T. (2003) Morphologically distinct microtubule ends in the mitotic centrosome of Caenorhabditis elegans. J. Cell Biol. 163, 451-456

20 Austin, J. R., 2nd, Segui-Simarro, J. M. and Staehelin, L. A. (2005) Quantitative analysis of changes in spatial distribution and plus-end geometry of microtubules involved in plant-cell cytokinesis. J. Cell Sci. 118, 3895-3903

21 Hoog, J. L., Schwartz, C., Noon, A. T., O'Toole, E. T., Mastronarde, D. N., McIntosh, J. R. and Antony, C. (2007) Organization of interphase microtubules in fission yeast analyzed by electron tomography. Dev. Cell 12, 349-361 
22 Koning, R. I., Zovko, S., Barcena, M., Oostergetel, G. T., Koerten, H. K., Galjart, N., Koster, A. J. and Mieke Mommaas, A. (2008) Cryo electron tomography of vitrified fibroblasts: microtubule plus ends in situ. J. Struct. Biol. 161, 459-468

23 Zovko, S., Abrahams, J. P., Koster, A. J., Galjart, N. and Mommaas, A. M. (2008) Microtubule plus-end conformations and dynamics in the periphery of interphase mouse fibroblasts. Mol. Biol. Cell 19, 3138-3146

24 Jánosi, I. M., Chrétien, D. and Flyvbjerg, H. (1998) Modeling elastic properties of microtubule tips and walls. Eur. Biophys. J. 27, 501-513

25 Job, D., Valiron, O. and Oakley, B. (2003) Microtubule nucleation. Current opinion in cell biology 15, 111-117

26 Hyman, A. A., Salser, S., Drechsel, D. N., Unwin, N. and Mitchison, T. J. (1992) Role of GTP hydrolysis in microtubule dynamics: information from a slowly hydrolyzable analogue, GMPCPP. Mol. Biol. Cell 3, 1155-1167.

27 Mitchison, T. and Kirschner, M. (1984) Dynamic instability of microtubule growth. Nature 312, 237-242.

28 Nogales, E., Whittaker, M., Milligan, R. A. and Downing, K. H. (1999) High-resolution model of the microtubule. Cell 96, 79-88

29 Chrétien, D., Jánosi, I., Taveau, J. C. and Flyvbjerg, H. (1999) Microtubule's conformational cap. Cell Struct. Funct. 24, 299-303

30 Buey, R. M., Diaz, J. F. and Andreu, J. M. (2006) The nucleotide switch of tubulin and microtubule assembly: a polymerization-driven structural change. Biochemistry 45, 59335938

31 Rice, L. M., Montabana, E. A. and Agard, D. A. (2008) The lattice as allosteric effector: structural studies of alphabeta- and gamma-tubulin clarify the role of GTP in microtubule assembly. Proc. Natl. Acad. Sci. U. S. A. 105, 5378-5383

32 Gebremichael, Y., Chu, J. W. and Voth, G. A. (2008) Intrinsic bending and structural rearrangement of tubulin dimer: molecular dynamics simulations and coarse-grained analysis. Biophys. J. 95, 2487-2499

33 Dimitrov, A., Quesnoit, M., Moutel, S., Cantaloube, I., Pous, C. and Perez, F. (2008) Detection of GTP-tubulin conformation in vivo reveals a role for GTP remnants in microtubule rescues. Science 322, 1353-1356

34 Amos, L. A. and Schlieper, D. (2005) Microtubules and maps. Adv. Protein Chem. 71, 257298

35 Moores, C. A., Perderiset, M., Francis, F., Chelly, J., Houdusse, A. and Milligan, R. A. (2004) Mechanism of microtubule stabilization by doublecortin. Mol. Cell 14, 833-839

36 Moores, C. A., Perderiset, M., Kappeler, C., Kain, S., Drummond, D., Perkins, S. J., Chelly, J., Cross, R., Houdusse, A. and Francis, F. (2006) Distinct roles of doublecortin modulating the microtubule cytoskeleton. EMBO J. 25, 4448-4457

37 Akhmanova, A. and Steinmetz, M. O. (2008) Tracking the ends: a dynamic protein network controls the fate of microtubule tips. Nat. Rev. Mol. Cell Biol. 9, 309-322

38 Lansbergen, G. and Akhmanova, A. (2006) Microtubule plus end: a hub of cellular activities. Traffic (Copenhagen, Denmark) 7, 499-507

39 Morrison, E. E. (2007) Action and interactions at microtubule ends. Cell. Mol. Life Sci. 64, 307-317

40 Bieling, P., Kandels-Lewis, S., Telley, I. A., van Dijk, J., Janke, C. and Surrey, T. (2008) CLIP-170 tracks growing microtubule ends by dynamically recognizing composite EB1/tubulin-binding sites. J. Cell Biol. 183, 1223-1233

41 Dixit, R., Barnett, B., Lazarus, J. E., Tokito, M., Goldman, Y. E. and Holzbaur, E. L. (2009) Microtubule plus-end tracking by CLIP-170 requires EB1. Proc. Natl. Acad. Sci. U. S. A. 106, 492-497 
42 Bieling, P., Laan, L., Schek, H., Munteanu, E. L., Sandblad, L., Dogterom, M., Brunner, D. and Surrey, T. (2007) Reconstitution of a microtubule plus-end tracking system in vitro. Nature 450, 1100-1105

43 Tirnauer, J. S., Grego, S., Salmon, E. D. and Mitchison, T. J. (2002) EB1-microtubule interactions in Xenopus egg extracts: role of EB1 in microtubule stabilization and mechanisms of targeting to microtubules. Mol. Biol. Cell 13, 3614-3626

44 Ligon, L. A., Shelly, S. S., Tokito, M. K. and Holzbaur, E. L. (2006) Microtubule binding proteins CLIP-170, EB1, and p150Glued form distinct plus-end complexes. FEBS Lett. 580, $1327-1332$

45 Sandblad, L., Busch, K. E., Tittmann, P., Gross, H., Brunner, D. and Hoenger, A. (2006) The Schizosaccharomyces pombe EB1 Homolog Mal3p Binds and Stabilizes the Microtubule Lattice Seam. Cell 127, 1415-1424

46 Slep, K. C. and Vale, R. D. (2007) Structural Basis of Microtubule Plus End Tracking by XMAP215, CLIP-170, and EB1. Mol. Cell 27, 976-991

47 Tirnauer, J. S., O'Toole, E., Berrueta, L., Bierer, B. E. and Pellman, D. (1999) Yeast Bim1p promotes the G1-specific dynamics of microtubules. J. Cell Biol. 145, 993-1007

48 Rogers, S. L., Rogers, G. C., Sharp, D. J. and Vale, R. D. (2002) Drosophila EB1 is important for proper assembly, dynamics, and positioning of the mitotic spindle. J. Cell Biol. 158, 873-884.

49 Busch, K. E. and Brunner, D. (2004) The microtubule plus end-tracking proteins mal3p and tip1p cooperate for cell-end targeting of interphase microtubules. Curr. Biol. 14, 548-559

50 Komarova, Y., De Groot, C. O., Grigoriev, I., Gouveia, S. M., Munteanu, E. L., Schober, J. M., Honnappa, S., Buey, R. M., Hoogenraad, C. C., Dogterom, M., Borisy, G. G., Steinmetz, M. O. and Akhmanova, A. (2009) Mammalian end binding proteins control persistent microtubule growth. J. Cell Biol. 184, 691-706

51 Ligon, L. A., Shelly, S. S., Tokito, M. and Holzbaur, E. L. (2003) The microtubule plus-end proteins EB1 and dynactin have differential effects on microtubule polymerization. Mol. Biol. Cell 14, 1405-1417

52 Manna, T., Honnappa, S., Steinmetz, M. O. and Wilson, L. (2008) Suppression of Microtubule Dynamic Instability by the +TIP Protein EB1 and Its Modulation by the CAPGly Domain of p150(Glued). Biochemistry 47, 779-786

53 Vitre, B., Coquelle, F. M., Heichette, C., Garnier, C., Chretien, D. and Arnal, I. (2008) EB1 regulates microtubule dynamics and tubulin sheet closure in vitro. Nature cell biology $\mathbf{1 0}$, 415-421

54 Nakamura, M., Zhou, X. Z. and Lu, K. P. (2001) Critical role for the EB1 and APC interaction in the regulation of microtubule polymerization. Curr. Biol. 11, 1062-1067.

55 Hayashi, I., Wilde, A., Mal, T. K. and Ikura, M. (2005) Structural basis for the activation of microtubule assembly by the EB1 and p150Glued complex. Mol. Cell 19, 449-460

56 Wolff, J., Sackett, D. L. and Knipling, L. (1996) Cation selective promotion of tubulin polymerization by alkali metal chlorides. Protein Sci. 5, 2020-2028

57 des Georges, A., Katsuki, M., Drummond, D. R., Osei, M., Cross, R. A. and Amos, L. A. (2008) Mal3, the Schizosaccharomyces pombe homolog of EB1, changes the microtubule lattice. Nat. Struct. Mol. Biol. 15, 1102-1108 


\section{Figure legends:}

Figure 1: Organization of the microtubule lattice. A) Lattice of a microtubule with 13 protofilaments (pfs) and 3 lateral helices (lhs) (denoted 13_3 microtubule). Pfs are parallel to the microtubule longitudinal axis. B) Lattice accommodation of a microtubule with 15 pfs and 4 lateral helices (15_4 microtubule). Pfs are skewed by a small angle $(\theta))$ and describe long-pitch helices around the microtubule wall. The red line highlights one lateral helix, whose continuity is maintained owing to the protofilament skewing. C) Sheets display two opposite curvatures resulting from the longitudinal intrinsic curvature of protofilaments (green arrow) and the lateral interactions between protofilaments (red arrow). The latter increases with the number of protofilaments, which leads to the gradual straightening and closure of the sheet into a cylinder (blue arrow). D) Two curved tubulin sheets observed by cryo-electron microscopy (arrow heads).

Figure 2: Alternative models to account for EB1/Mal3p effects on microtubule structure and dynamics. (A) EB1/Mal3p (in red) would bind to the sheet edges and accelerate tube closure either through a zippering activity or an increase of the sheet lateral growth (see the text for details). Both models result in the reduction of the conformational cap thought to stabilize microtubules, making the polymers more prone to undergo a catastrophe. Such a mechanism would indirectly favor the selection of the most stable 13_3 microtubules. (B) EB1 forces the tube to close into 13_3 configurations, probably by controlling lateral interactions between adjacent tubulin dimers in the area around the tube junction. Inspired from [45, 53, 57]. 


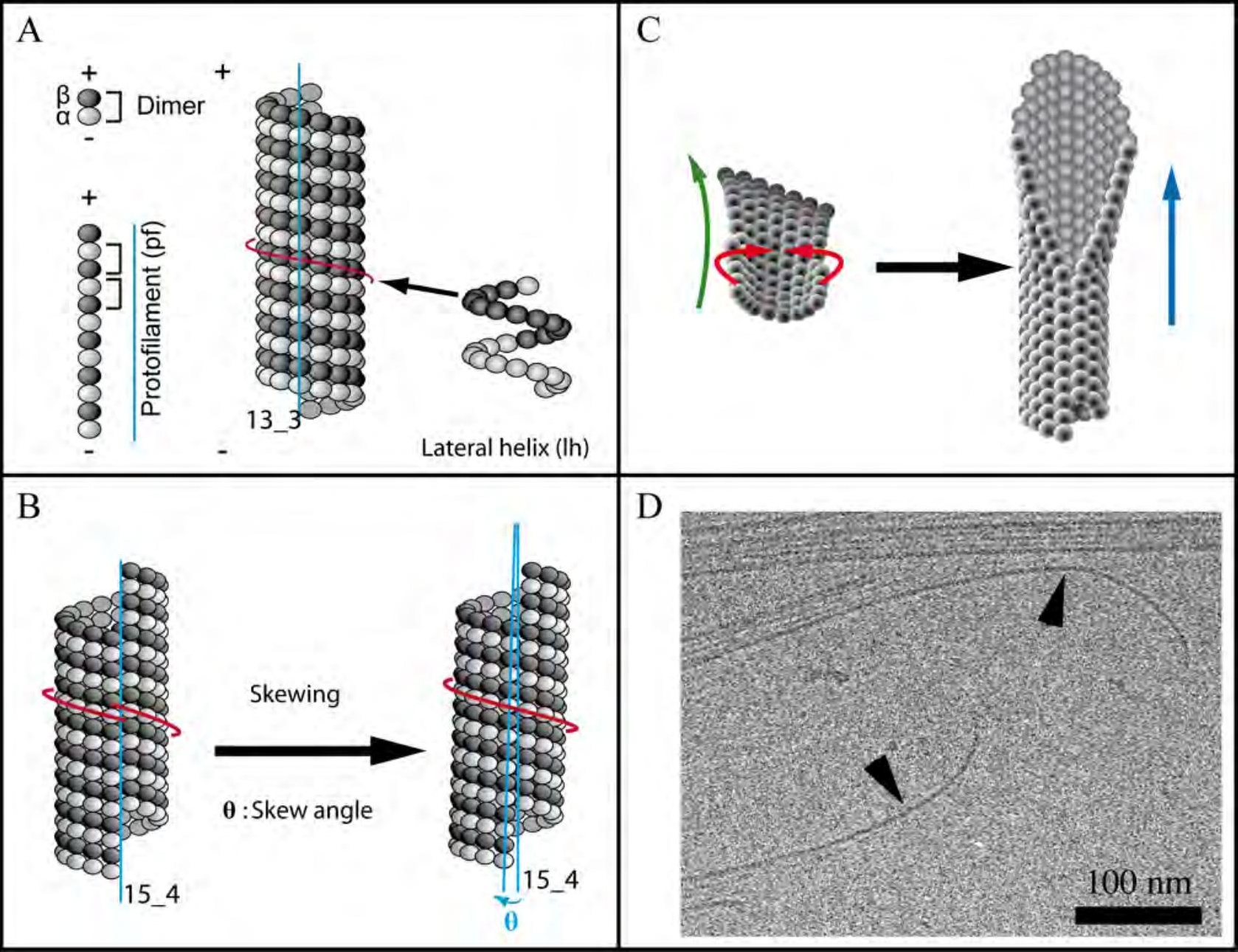

A $\left.{ }_{\alpha}^{\beta} \stackrel{+}{8}\right]$ Dimer

$8=\left.8_{8}^{+}\right|^{\mid \frac{4}{2}}$

Lateral helix (Ih)

\section{B} 15_4 
